\title{
Modulation of Neuritogenesis by a Protein Implicated in X-Linked Mental Retardation
}

\author{
Xinfu Jiao, Hongxin Chen, Jianmin Chen, Karl Herrup, Bonnie L. Firestein, and Megerditch Kiledjian \\ Department of Cell Biology and Neuroscience, Rutgers University, Piscataway, New Jersey 08854-8082
}

Posttranscriptional regulation is an important control mechanism governing gene expression in neurons. We recently demonstrated that VCX-A, a protein implicated in X-linked mental retardation, is an RNA-binding protein that specifically binds the $5^{\prime}$ end of capped mRNAs to prevent their decapping and decay. Previously, expression of VCX-A was reported to be testes restricted. Consistent with a role in cognitive function, we demonstrate that VCX-A is ubiquitously expressed in human tissues including the brain. Moreover, retinoic acid-induced differentiation of human SH-SY5Y neuroblastoma cells promoted the accumulation of VCX-A in distinct cytoplasmic foci within neurites that colocalize with staufen1-containing RNA granules, suggesting a role in translational suppression and/or mRNA transport. Exogenous expression of VCX-A in rat primary hippocampal neurons, which normally do not express the primate-restricted VCX proteins, promoted neurite arborization, and shRNA-directed knockdown of the VCX genes in SH-SY5Y cells resulted in a reduction of both primary and secondary neurite projections upon differentiation. We propose that the cap-binding property of VCX-A reflects a role of this protein in mRNA translational regulation. In support of this hypothesized role, we demonstrate that VCX-A can specifically bind a subset of mRNAs involved in neuritogenesis and is also capable of promoting translational silencing. Thus, VCX-A contains the capacity to modulate the stability and translation of a subset of target mRNAs involved in neuronal differentiation and arborization. It is plausible that defects of these functions in the absence of the VCX genes could contribute to a mental retardation phenotype.

\section{Introduction}

The regulation of gene expression has a fundamental impact on the development, differentiation, and proliferation of cells and can occur at multiple levels including mRNA metabolism. An important property of all mRNAs is their inherent stability and their capacity to be translated into protein. In eukaryotes, the $5^{\prime}$ cap and $3^{\prime}$ polyadenylated $[\operatorname{poly}(\mathrm{A})]$ tail are critical structures for mRNA stability and translation. Following removal of their poly(A) tail, mRNAs are primarily degraded by two exonucleolytic pathways involving either continual decay from the $3^{\prime}$ end or a decapping step preceding 5' to $3^{\prime}$ exonucleolytic decay (Liu and Kiledjian, 2006; Garneau et al., 2007). Removal of the cap renders the mRNA translationally incompetent since the cap is required for ribosomal recruitment and the exposed 5' end is susceptible to exoribonucleolytic decay.

We recently identified VCX-A as a regulator of mRNA stability and showed that it can function as an inhibitor of the Dcp2 mRNA decapping enzyme (Jiao et al., 2006). VCX-A is a member of a family of proteins encoded by distinct genes located on the $\mathrm{X}$ chromosome (Xp22), termed VCX-A, VCX-B, VCX-B1, and

Received Dec. 15, 2008; revised Aug. 7, 2009; accepted Aug. 27, 2009.

These studies were supported by National Institutes of Health (NIH) Grants AG024494 and NS20591 to K.H. National Science Foundation Grant IBN-0548543, March of Dimes Grant 1-FY08-464, and a National Alliance for Research on Schizophrenia and Depression Toulmin Independent Investigator Award to B.L.F., and NIH Grant GM67005 to M.K. We thank Terri G. Kinzy and Anthony M. Esposito for assistance with the polysome gradient experiments.

Correspondence should be addressed to Megerditch Kiledjian, Department of Cell Biology and Neuroscience, Rutgers University, 604 Allison Road, Piscataway, NJ 08854-8082. E-mail: kiledjian@biology.rutgers.

DOI:10.1523/JNEUROSCI.5954-08.2009

Copyright $\odot 2009$ Society for Neuroscience ～0270-6474/09/2912419-09\$15.00/0
VCX-C, as well as two identical copies on the Y chromosome, termed VCY. The proteins encoded by the different VCX/Y genes have a $>95 \%$ identity at the $\mathrm{N}$-terminal half of the protein and primarily differ in the number of 10 aa repeats at their $\mathrm{C}$ termini. Interestingly, the VCX-A gene was identified as the defining interval in patients with X-linked mental retardation (MRX) (Fukami et al., 2000). However, subsequent studies indicated that the potential role of the VCX proteins in MRX is probably more complex (Van Esch et al., 2005; Hosomi et al., 2007; Macarov et al., 2007; Cuevas-Covarrubias and Gonzalez-Huerta, 2008) and likely involves a cumulative VCX dosage rather than reflecting the output of the VCX-A gene alone (Van Esch et al., 2005).

In the mammalian neurons, transcription and translation are often uncoupled in time and space, and this autonomous control of gene expression can play a critical role in the enduring (late-stage) form of synaptic plasticity thought to underlie learning and memory (Frey et al., 1988; Nguyen et al., 1994). Various strategies for the regulation of protein translation are used in neurons to control local protein synthesis in dendrites (for review, see Sutton and Schuman, 2005). These regulatory processes are involved in mRNA localization, translation, and degradation. In this context, it is not surprising to find that several RNA-binding proteins are known to contribute to synaptic plasticity through their regulation of specific mRNA subsets. These include FMRP, CPEB (cytoplasmic polyadenylation element binding factor), and Nova (neuro-oncological ventral antigen) (Wu et al., 1998; Huber et al., 2002; Si et al., 2003; Alarcon et al., 2004; Huang et al., 2005; Koekkoek et al., 2005). In this report, we demonstrate that the VCX-A gene product is a translational inhibitor and is involved in the regulation of neuronal cell morphogenesis and neuritogenesis. 


\section{Materials and Methods}

Plasmids and expression of recombinant protein. The pET28a-VCX-A, pET28a-VCX-A ${ }^{\Delta N 40}$, pcDNA3-FLAG-VCX-A, pcDNA3-FLAG-VCX$\mathrm{A}^{\Delta \mathrm{N} 40}$, and pIRESpuro3-VCX-A plasmids have been described previously (Jiao et al., 2006), as has the pSV2AL $\Delta 5^{\prime}$ plasmid (de Wet et al., 1987). The pIRESpuro3-VCX-A plasmid expressing myc-tagged VCX-A was used to generate the VCX-A-expressing stable cell line. pET28a-UlP1 and pcDNA3-FLAG-IKK plasmids were kindly provided by C. D. Lima (Sloan-Kettering Institute, New York, NY) and G. Xiao (University of Pittsburgh, Pittsburgh, PA), respectively. The two hairpin-containing VCX sequences were generated according to Paddison and Hannon (2002). The annealed DNA oligonucleotides were inserted into the BseR1 and BamHI sites of the pSHAG-1 vector to generated PSHAG-VCX-A. The pSHAG-1 vector contains a U6 promoter shRNA expression cassette and was kindly provided by G. Hannon (Cold Spring Harbor Laboratory, Cold Spring Harbor, NY). The generated hairpin RNA targets the VCX mRNA sequence, 5' CAC TGA GTC AGG AGA GCG AGG TGG AAG AA 3' (VCX-A 645-673 bp and 705-733 bp).

GST fusion and His-tagged proteins were expressed and purified according to the manufacturers' instructions (GE Healthcare and Novagen, respectively) with minor modification as previously described (Jiao et al., 2006).

Cell culture, transfection, and stable cell line generation. Human K562 erythroleukemia cells, HeLa epithelial carcinoma cells, 293T embryonic kidney cells, SH-SY5Y neuroblastoma cells, mouse NIH 3T3 embryonic fibroblast cells, and MEL mouse erythroleukemia cells were obtained from ATCC and grown according to the supplier. All transfections were performed using Lipofectamine 2000 (Invitrogen) according to the manufacturer's protocol. 293T cells expressing a stably integrated VCX-A gene or VCX-A ${ }^{\Delta N 40}$ mutant were generated by transfecting $293 \mathrm{~T}$ cells with pIRESpuro3-VCX-A, VCX-A ${ }^{\triangle \mathrm{N} 40}$ mutant, or pIRESpuro3 vector plasmids and selected with $3 \mu \mathrm{g} / \mathrm{ml}$ puromycin (Sigma-Aldrich) as described previously (Jiao et al., 2006). Serial dilutions were performed to isolate a monoclonal cell line expressing high levels of VCX-A. Transcriptional arrest experiments were performed by treating 293T embryonic kidney cells stably expressing myc-tagged VCX-A, VCX-A ${ }^{\Delta \mathrm{N} 40}$ mutant, or vector control with $5 \mu \mathrm{g} / \mathrm{ml}$ actinomycin D for $0,0.5,1,2$, or $3 \mathrm{~h}$.

Neuronal differentiation of the human neuroblastoma SH-SY5Y cell line was induced by the addition of $10 \mu \mathrm{M}$ retinoic acid (RA; SigmaAldrich) to the culture media for $2 \mathrm{~d}$. The differentiation phenotype of SH-SY5Y cells with RA treatment was confirmed by photography under a phase contrast microscope (Zeiss Axiovert $100 \mathrm{M}$ microscope) to follow neurite generation.

Extract preparation. Human tissues were obtained from the Cancer Institute of New Jersey. Tissue extracts and cell extracts were prepared by sonication as previously described (Rodgers et al., 2002) in PBS buffer containing protease inhibitors (Complete Protease Inhibitor Cocktail Tablets; Roche).

RNA generation. The ${ }^{32} \mathrm{P}$-cap-labeled pcP RNA was generated as previously described (Wang et al., 1999, 2002). The Firefly luciferase reporter gene was PCR amplified from pSV2AL $\Delta 5^{\prime}$ with a $5^{\prime}$-specific reporter gene primer containing a T7 promoter primer adaptor (5'-CGTAATACGACTCACTATAGGGCATTCCGGTACTGTTGGTAAAATGG-3') and a $3^{\prime}$-specific reporter gene primer containing an $\mathrm{A}_{60}$ tail $\left(5^{\prime}-\mathrm{T}_{60}\right.$ GCCGCCCACTCAGACTTTATTCAAAGACC- $\left.3^{\prime}\right)$. The PCR product was used as template to transcribe RNA with T7 RNA polymerase (Promega). The cap analog $\mathrm{m}^{7} \mathrm{GpppG}$ was added to the in vitro transcription system to generate capped-Firefly luciferase RNA. The generated polyadenylated luciferase reporter mRNA was used as substrate for protein translation.

VCX antibody generation and affinity purification. The antiserum to VCX was commercially generated (Cocalico Biologicals) by immunizing rabbits with recombinant His-VCX-A. The polyclonal sera was affinity purified with GST-VCX-A fusion protein consisting of the C-terminal 105 aa of VCX-A coupled to an NHS column according to the manufacturer's instructions (GE Healthcare Life Science).

Western blot, reverse transcriptase-PCR, and quantitative real-time $P C R$ analysis. Cell or tissue extracts were resolved by $12.5 \%$ SDS-PAGE.
VCX-A C-terminal affinity-purified polyclonal antibody (1:200 dilution), monoclonal anti-eIF4E antibody (1:1500 dilution; Transduction Laboratories), polyclonal anti-CaMKI antibody (1:5000 dilution; Abcam), and monoclonal anti-GAPDH antibody (1:2000 dilution; Abcam) were used for Western blot analysis and visualized using secondary antibodies coupled to horseradish peroxidase (Jackson ImmunoResearch) and chemiluminescence (ECL; GE Healthcare Life Science).

Total RNA was isolated from human tissues and cell lines using Trizol (Invitrogen) and subjected to RQ1 RNase-Free DNase (Promega) treatment to prevent genomic DNA contamination. The RNAs were reverse transcribed into cDNA with Oligo $\mathrm{d}(\mathrm{T})$ primer using the Superscript II reverse-transcription kit according to the manufacturer (Invitrogen). PCR amplification with the forward primer 5'-ATG AGT CCA AAG CCG AGA GC-3' and reverse primer 5' ${ }^{\prime}$-TCA CGG CCG CCA TCT TTG TC-3' results in the amplification of all members of the VCX/Y gene family and generates a $187 \mathrm{bp}$ fragment. VCY was amplified by the VCYspecific primers 5'-ATG AGT CCA AAG CCG AGA GCC TC-3' and 5'-TGA CTC AGT GGT TCT TCC ACC TC-3' to generate a 451 bp fragment. Reverse transcription products $(4 \mu \mathrm{l})$ were used as PCR templates and amplified for 40 cycles. The PCR products were resolved by a $2.5 \%$ agarose gel.

The stability of electroporated firefly luciferase mRNA and endogenous CaMKI mRNA were determined in 293T cells stably expressing myc-tagged VCX-A, VCX-A ${ }^{\Delta \mathrm{N} 40}$ truncation, or vector control by quantitative real-time PCR. Transcription was block by the addition of 5 $\mu \mathrm{g} / \mathrm{ml}$ actinomycin D to the cell culture medium. GAPDH mRNA was used as an internal control. Real-time PCR was performed using SYBR green PCR core reagent (Applied Biosystems) according to the manufacturer's instructions. Data were analyzed by the comparative Ct method as previously described (Livak and Schmittgen, 2001).

Sequences for all primer sets are listed in supplemental Table 1 (available at www.jneurosci.org as supplemental material).

Immunohistochemistry assay. Formalin-fixed paraffin-embedded 10 $\mu \mathrm{m}$ human brain sections were obtained from the Alzheimer Disease Research Center at Case Western Reserve University. Immunohistochemistry assay was performed as described previously (Busser et al., 1998). Briefly, deparaffinized sections were treated with citrate buffer to retrieve antigen, and then soaked in $0.3 \%$ hydrogen peroxide to remove endogenous peroxidase activity. Affinity-purified VCX-specific antibody was diluted in blocking solution (1:100) and used to detect VCX in the tissue and detected with biotinylated goat anti-rabbit secondary antibody (1:400), avidin-biotin complex horseradish peroxidase, and DAB substrate (Vector Laboratories). Sections were counterstained with hematoxylin to visualize nuclei.

Immunofluorescence assay. SH-SY5Y cells were grown on poly-lysinecoated glass coverslips in six-well plates to $70 \%$ confluency. The coverslips containing the cells were treated as previously described (Liu et al., 2004). Affinity-purified VCX-A C-terminal rabbit polyclonal antibody was used as primary antibodies to detect endogenous VCX and monoclonal anti-staul antibody (1:80 dilution; Sigma-Aldrich) was used to detect staufen1-containing neuronal RNA granules. Monoclonal antineuron-specific class III $\beta$-tubulin (TuJ1) antibody (Abcam) (1:1000 dilution) was used as a neuronal marker. A goat anti-rabbit or goat anti-mouse secondary antibody conjugated with Texas Red or FITC (Jackson ImmunoResearch) was used as noted in the figure legends at 1:200 dilutions. The nuclei were stained with $1 \mu \mathrm{g} / \mathrm{ml}$ DAPI for $3 \mathrm{~min}$. The images were obtained with a Zeiss Axiovert $100 \mathrm{M}$ microscope.

Electroporation and luciferase activity assay. The in vitro transcribed capped-Firefly luciferase mRNA ( $1 \mu \mathrm{g})$ was electroporated into 293T cells in Opti-MEM I reduced-serum medium (Invitrogen) in a $4 \mathrm{~mm}$ gap cuvette using a Bio-Rad Genepulser charged at $320 \mathrm{~V}, 250 \mu \mathrm{F}$. All reagents and cuvettes were kept on ice before electroporation. Following the discharge, the cells were washed once with $\mathrm{PBS}\left(25^{\circ} \mathrm{C}\right)$ and immediately resuspended in prewarmed D-MEM $/ 10 \% \mathrm{FBS}$ medium at $37^{\circ} \mathrm{C}$ and aliquoted into different plates. The cells were harvested at the indicated time points to detect luciferase activity.

Firefly luciferase activity was detected using a Luciferase Assay System (Promega) according to the manufacturer's guidelines using GloMax-Multi Luminescence Module (Promega). 

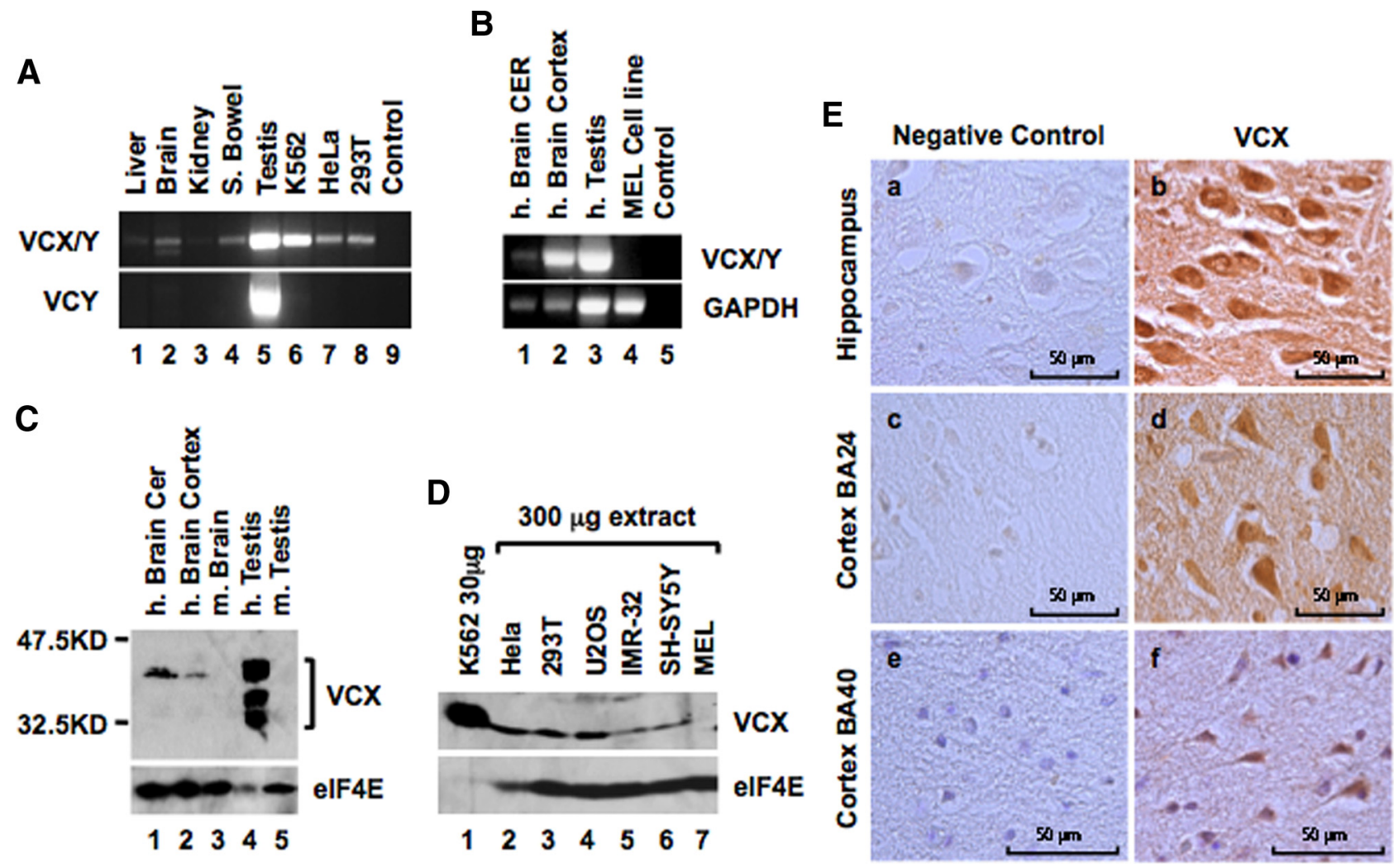

Figure 1. VCX is ubiquitously expressed in human tissues and cell lines. $A, B$, Total RNA was isolated from the indicated human tissues and cell lines, reverse transcribed with oligo $d(T)$, and PCR amplified with a primer set that recognizes all the VCX and VCY genes as well as a set that was specific for VCY as denoted. GAPDH was used as a RNA loading control in $B$. All cells, tissues are of human origin except MEL mouse erythroleukemia cells. $C, D$, Total extract from the indicated human and mouse tissues and cell lines were separated by SDS-PAGE and the presence of VCX proteins detected by Western blot analysis using affinity-purified rabbit anti-VCX antisera. Migration of the VCX protein is shown on the right and protein size markers are indicated on the left. The multiple bands in the testis extract are likely due to the high level of expression of all the VCX and VCY family proteins that are expressed in this tissue resulting in dimers and/or aggregates in the gel. Samples in C with an " $\mathrm{h}$ " prefix are of human origin and " $\mathrm{m}$ " are of mouse. All lanes contain $150 \mu \mathrm{g}$ of total protein except the human testes, which contains $60 \mu \mathrm{g}$. Samples in D are all from human origin except MEL cells and protein amounts are as indicated. $\boldsymbol{E}$, Postmortem $10 \mu \mathrm{m}$ human hippocampus and cortex Brodmann area (BA) 24 (anterior cingulate cortex) and 40 (supramarginal gyrus) brain sections are shown. Immunohistochemistry was performed in the presence of affinity-purified polyclonal VCX-specific primary antibody $(\boldsymbol{b}, \boldsymbol{d}, \boldsymbol{f})$ or in its absence $(\boldsymbol{a}, \boldsymbol{c}, \boldsymbol{e})$. Nuclei were stained with DAPI (blue) $(e, f)$. Images of $400 \times$ magnification are shown with clear VCX expression in neurons and neuropil. Scale bars are as indicated.

mRNA coimmunopurification assay. Anti-Flag monoclonal antibody coupled to M2 agarose beads (Sigma-Aldrich) were used for the coimmunopurification assays. FLAG-tagged VCX-A or mDAZL were transiently overexpressed in human SH-SY5Y neuroblastoma cells for $48 \mathrm{~h}$ and differentiated with $10 \mu \mathrm{M}$ RA. SH-SY5Y cells transfected with the FLAG-tagged empty vector were used as a negative control. Anti-Flag M2 agarose beads $(30 \mu \mathrm{l})$ were incubated with $1 \mathrm{mg}$ of cell extract expressing FLAG-VCX-A or FLAG-mDAZL at $4^{\circ} \mathrm{C}$ for $1 \mathrm{~h}$ in PBS with moderate shaking in the presence of $1 \times$ protease inhibitor cocktail (Roche) and RNase inhibitor (1:50 dilution; Promega). Following three washes with $\mathrm{PBS} / 250 \mathrm{~mm} \mathrm{NaCl} / 300 \mathrm{~mm}$ urea/25\% Triton X-100, the coimmunopurified mRNAs were eluted from the M2 agarose beads with TE/1\% SDS and oligo $\mathrm{d}(\mathrm{T})$ reverse transcribed and PCR amplified with transcriptspecific primers (listed in supplemental Table 1, available at www. jneurosci.org as supplemental material) and PCR products resolved on a $2.5 \%$ agarose gel. SH-SY5Y total RNA reverse transcriptase (RT)-PCR was used as a positive control for the PCRs.

Polysome profile and Northern blotting. 293T VCX-A stable cells and $293 \mathrm{~T}$ vector control cells were transiently transfected with the firefly luciferase reporter plasmid pSV2AL $\Delta 5^{\prime}$. Cells were cultured at $37^{\circ} \mathrm{C}$ for $16 \mathrm{~h}$ to $\sim 60-70 \%$ confluency. Cycloheximide $(100 \mu \mathrm{g} / \mathrm{ml})$ was added to the medium, and cells were cultured for $15 \mathrm{~min}$ followed by washing with ice-cold PBS containing cycloheximide. The cells were harvested and lysed with ice-cold polysome fraction lysis buffer $[0.15 \mathrm{M} \mathrm{KCl}, 15 \mathrm{~mm}$ MgOAc, $20 \mathrm{~mm}$ Tris-HCl, $\mathrm{pH}$ 7.5, $1 \mathrm{mg} / \mathrm{ml}$ heparin, $1 \mathrm{~mm}$ DTT, 100 $\mu \mathrm{g} / \mathrm{ml}$ cycloheximide, $0.5 \%$ Triton $\mathrm{X}-100,1 \times$ protease inhibitor cocktail (Roche), and RNase inhibitor (1:50 dilution; Promega)]. Cell extracts were spun at $10,000 \times g$ for $10 \mathrm{~min}$ at $4^{\circ} \mathrm{C}$. The supernatant was trans- ferred to a fresh tube and $\mathrm{OD}_{260}$ was measured. Cell extracts $(100 \times$ $\mathrm{OD}_{260}$ ) were layered on $12 \mathrm{ml} 10-50 \%$ sucrose gradients and centrifuged for $4 \mathrm{~h}$ at $23,000 \mathrm{rpm}$ in a Sorvall Superspin 600 rotor at $4^{\circ} \mathrm{C}$. $\mathrm{A}_{254}$ was monitored using a Model 185 density gradient fractionator (ISCO) and each $1 \mathrm{ml}$ gradient fraction was collected.

Protein aliquots from the gradients were precipitated with trichloroacetic acid (TCA) and resolved by $12.5 \%$ SDS-PAGE for Western blot analysis and detected with polyclonal anti-c-Myc antibody (1:500 dilution, Santa Cruz Biotechnology). For Northern blot assay, the RNAs were isolated with $1 \%$ SDS and $10 \mathrm{~mm}$ EDTA, precipitated with isopropanol after phenol/chloroform extraction and resolved on a $1 \%$ formaldehyde denaturing agarose gel. RNA was transferred to a Hybond-N member (GE Healthcare Life Science), and cross-linked to membrane at $80^{\circ} \mathrm{C}$ under vacuum for $1 \mathrm{~h}$. DNA-specific probes were labeled with $\left[\alpha-{ }^{32} \mathrm{P}\right] \mathrm{dCTP}$ by PCR. The hybridization was performed at $60^{\circ} \mathrm{C}$ overnight and washed with $0.1 \%$ SSC/0.1\% SDS. Quantifications were performed using a Molecular Dynamics PhosphorImager (Storm860) using ImageQuant-5 software.

Rat primary neuron culture, transfection, and assessment of neurite number. Primary neuronal cultures at a density of $\sim 1800$ cells $/ \mathrm{mm}^{2}$ were prepared from hippocampi of rat embryos at $18 \mathrm{~d}$ gestation and grown as previously described (Firestein et al., 1999; Chen and Firestein, 2007). After $10 \mathrm{~d}$ in vitro (DIV), neurons were transfected with plasmids encoding FLAG-tagged VCX-A or VCX-A ${ }^{\Delta \mathrm{N} 40}$ using Effectene (Qiagen). FLAG-tagged empty vector plasmid was used as a control. pEGFP-C1 (BD Biosciences) was cotransfected to enable detection and visualization of transfected cells. Forty-eight hours after transfection, neurons were fixed in $4 \%$ paraformaldehyde in PBS for $15 \mathrm{~min}$ and immunostained 
using a monoclonal anti-FLAG tag antibody (Sigma-Aldrich) and polyclonal anti-GFP antibody (Abcam). The transfected neurons were identified and visualized by GFP fluorescence at $400 \times$ using Zeiss Axiovert $100 \mathrm{M}$ fluorescence microscope. Rat primary neuron neurite numbers and human SH-SY5Y neuroblastoma cell neurite numbers were counted from digitally obtained images of cells under experimentally blind conditions as previously described (Akum et al., 2004). Only those processes $>3$ $\mu \mathrm{m}$ were counted as neurites (Akum et al., 2004). Data were analyzed using GraphPad InStat software (Graph Pad) with ANOVA followed by the appropriate multiple-comparisons test or with Student's $t$ test when appropriate.

\section{Results}

The VCX genes are ubiquitously expressed in all human tissues, cell lines, and neurons tested

VCX gene expression was reported to be restricted to the testis (Fukami et al., 2000; Lahn and Page, 2000; Zou et al., 2003). Nevertheless, we recently reported the cloning of the VCX-A gene product from human K562 erythroleukemia cells (Jiao et al., 2006), suggesting perhaps a preferential, rather than exclusive, expression in the testis. To determine whether the VCX/Y genes are more ubiquitously expressed, we performed RT-PCR of different human and mouse tissues and cell lines using primers that correspond to conserved sequences at the $5^{\prime}$ end of all of the VCX/Y mRNAs. As shown in Figure 1, $A$ and $B$, VCX mRNAs can be detected in all human tissues and cell lines tested but not in the mouse erythroleukemia cell mRNA negative control (Fig. $1 B$, lane 4). Interestingly, the use of primers that exclusively detect the Y chromosome VCY gene products reveals that expression of this gene was restricted to testes (Fig. $1 A$, lane 5 ). To further expand upon the expression of the VCX genes, primers that specifically detect VCX, but not VCY, mRNA were used. As expected, VCX mRNA can be detected in all the human tissue and cell lines tested (supplemental Fig. S1, available at www. jneurosci.org as supplemental material).

To determine whether VCX proteins can be detected in tissues other than testes, we generated antibodies directed against the VCX protein and probed various human tissues for the presence of VCX. Western blot analysis revealed the presence of VCX in human testes extract (Fig. 1C, lane 4) as well as in human brain (Fig. 1C, lanes 1,2) but not in mouse brain and mouse testes (Fig. $1 C$, lanes 3 and 5, respectively). Expression in human brain was further confirmed with immunohistochemistry. Paraffin sections of human autopsy material probed with affinity-purified polyclonal VCX-specific antibody revealed a clear presence of the VCX proteins in neuronal cells of human brain (Fig. $1 E b, E d, E f$ ). The staining appeared equally robust in both neocortex and hippocampus. The cell bodies were strongly stained, but a significant level of immunoreactivity was found in the neuropil as well (Fig. $1 E$, compare $b, d$, and $f$ with $a, c$, and $e$ ). This is consistent with a function in both the perikaryon and proxi-
TuJ1
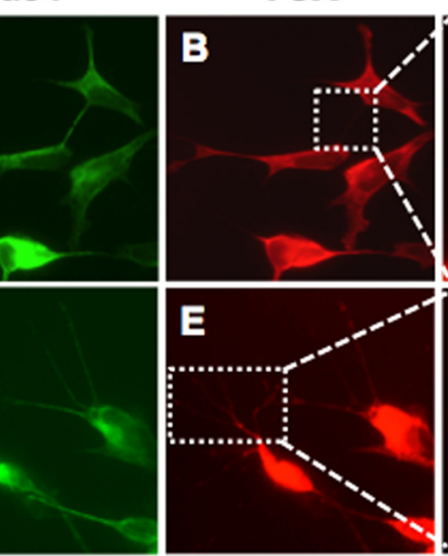

vcx

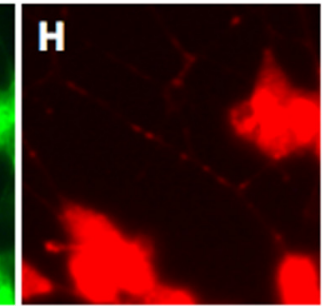

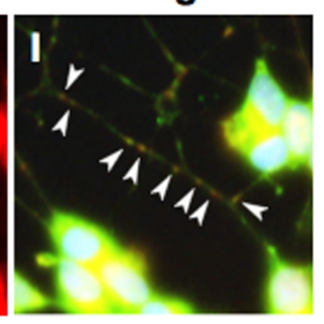

Expanded View

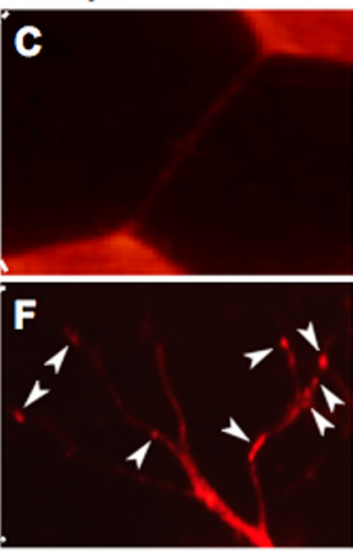

Merge
Figure 2. VCX foci number and size increase with differentiation of SH-SY5Y neuroblastoma cells. Human SH-SY5Y neuroblas oma cells were cultured in DMEM/F12 10\% FBS with $(\boldsymbol{D}-\boldsymbol{F})$ or without $(\boldsymbol{A}-\boldsymbol{C}) 10 \mu \mathrm{m}$ retinoic acid on poly-lysine-coated coverslips

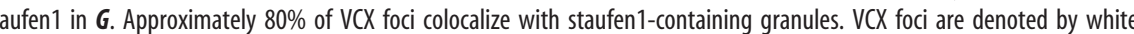

mal dendrites. The nucleus, though positive, was less darkly stained in most cells.

VCX protein is abundant in human K562 erythroleukemia cells (Fig. 1D, lane 1), and although at lower levels, is also detected in the transformed human cell lines tested (Fig. $1 D$, lanes 2-6) but not in mouse MEL cells (Fig. 1D, lane 7). Due to a $>92 \%$ identity between the different VCX and VCY proteins (Fukami et al., 2000; Lahn and Page, 2000), we are unable to unambiguously determine the identity of the multiple detected protein bands in testes. However, RT-PCR analysis revealed the expression of multiple VCX genes in all tested human tissues and cell lines (supplemental Fig. S1, available at www.jneurosci.org as supplemental material).

Collectively, our data are consistent with previous reports indicating that VCX/Y genes are restricted to simian primates but not prosimian or nonprimate mammals (Fukami et al., 2000; Lahn and Page, 2000). However, in contrast to these reports, our data indicate that only the VCY genes show testes-specific expression, while the VCX proteins are present in all tissues we have tested, including neurons of the CNS.

VCX protein expression and localization are associated with neuronal cell differentiation

VCX-A was initially identified as a candidate gene implicated in X-linked mental retardation (Fukami et al., 2000), suggesting a specific function in neurons. To determine a potential role for VCX-A in neuronal cells, we used the human SH-SY5Y neuroblastoma cell line to determine the fate of VCX upon neuronal 

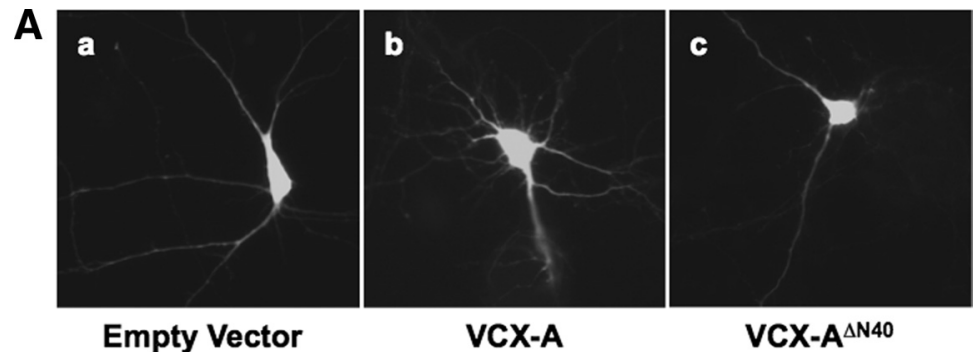

B

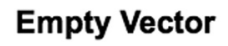

VCX-A

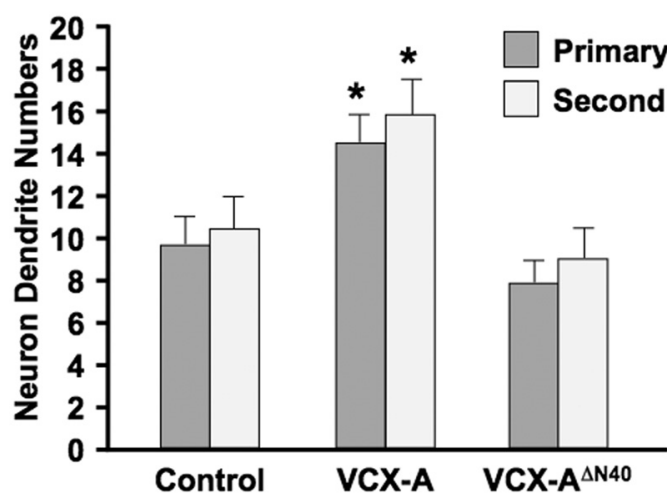

Figure 3. VCX-A affects neurite patterning in primary neurons. Neuronal cultures were prepared from hippocampi of rat embryos at $18 \mathrm{~d}$-gestation. $\boldsymbol{A}$, Representative neurons (GFP images shown) cotransfected using GFP plasmid with FLAG-tagged empty vector plasmid (a), FLAG-tagged VCX-A plasmid (b), or FLAG-tagged VCX-A ${ }^{\Delta N 40}$ plasmid (c). $\boldsymbol{B}$, Q Quantitation of the average number of primary and secondary neurites in neurons transfected with the FLAG empty control vector $(n=31)$, FLAG-tagged VCX-A $(n=25)$ and FLAG-tagged VCX-A ${ }^{\Delta N 40}(n=26)$ are shown. Expression of VCX-A protein increases primary (14.60 \pm 0.69$)$ and secondary ( $16.00 \pm 0.92)$ neurites compared with control neurons ( $9.97 \pm 0.68$ and $10.58 \pm 0.81$, respectively). Primary and secondary neurites increased 1.46- $(p<0.0001)$ and 1.51- $(p<0.0001)$ fold more than control neurons (ANOVA followed by Tukey-Kramer multiple-comparisons test). Overexpressed VCX-A ${ }^{\Delta \mathrm{N} 40}$ did not affect primary dendrites $(7.77 \pm 0.56, p>0.05)$ or secondary neurites $(9.08 \pm 0.73, p>0.05)$ compared with the control neurons. Where indicated, asterisks denote $p<0.0001$ by ANOVA followed by Tukey-Kramer multiple-comparisons test. $N$ values represent total number of cells counted and are derived from at least three independent transfections with \pm SD denoted by the error bars.

differentiation. Induction of SH-SY5Y cells with RA for $2 \mathrm{~d}$ promotes differentiation into a neuronal phenotype (Påhlman et al., 1984 ) with an increase of neurite projections (Fig. 2, compare $A$ with $D$ ). Although VCX protein levels were not detectably changed upon induced differentiation (data not shown), VCX foci number and size increased (Fig. 2E). In particular, VCX foci were prominent within the neurite projections (expanded view in Fig. $2 F$ ), suggesting a function in neuronal cell projections. A similar increase in VCX foci was also observed upon differentiation of human $\mathrm{H} 9$ neuronal stem cells (data not shown). These data suggest that VCX could be involved in neuronal process development and/or maintenance. Interestingly, the VCX foci did not colocalize with cytoplasmic processing $(\mathrm{P})$-bodies or stress granules (supplemental Fig. S2, available at www.jneurosci. org as supplemental material), indicating that they are distinct from these two well characterized cytoplasmic structures, but they do colocalize with staufen1-containing RNA granules (Fig. $2 G-I$ ), indicating they may be involved in mRNA transport or mRNA translational suppression (Kiebler and Bassell, 2006).

\section{VCX proteins affect proper neuritogenesis}

Normal cognitive function depends on proper connections between neurons. Abnormal morphogenesis and numbers of dendrites are associated with cognitive impairments, such as in Fragile X syndrome (McKinney et al., 2005; Grossman et al., 2006). Association of the VCX-A gene with MRX indicates that VCX-A, and likely other members of this family of proteins, may affect normal synaptogenesis or neurite numbers. The observed increase in accumulation of VCX protein into foci upon differentiation indicates that VCX may affect normal neuronal morphology. Since the VCX/Y genes are only present in primates and absent from lower mammals (Fukami et al., 2000; Lahn and Page, 2000) (Fig. 1), we used primary hippocampal neurons from rats as a model of a VCX-null system. Neurons were isolated from hippocampi of rat embryos at $18 \mathrm{~d}$ gestation and cotransfected with a plasmid expressing FLAGVCX-A and a plasmid expressing green fluorescent protein (GFP) as a marker to detect transfected cells (Akum et al., 2004). Upon expression of FLAG-VCX-A for $48 \mathrm{~h}$ following the initial transfection, the numbers of primary and secondary neurites both significantly increased compared with the control neurons (1.46- and 1.51-fold, respectively; $n=25, p<0.0001$ ) (Fig. $3 A, B)$. The increase was a consequence of VCX-A, since transfection of a construct expressing a mutant VCX-A protein lacking the first 40 aa that is unable to bind the $5^{\prime}$ cap (VCX-A ${ }^{\Delta \mathrm{N} 40}$ ) (Jiao et al., 2006) did not affect neurite branching $(n=26, p>0.05)$.

The above data indicate that overexpression of VCX-A in a cell that normally does not contain this protein can promote neurite arborization, suggesting that VCX-A is a positive regulator of neurite outgrowth. To further expand upon the role of VCX proteins, VCX levels were reduced in SH-SY5Y cells using a VCX-specific shRNA-directed knockdown (Fig. 4A; supplemental Fig. S3, available at www. jneurosci.org as supplemental material). The consequence on neurite outgrowth in SH-SY5Y cells upon VCX knockdown is shown in Figure $4 B$. Following transfection of either a control or VCX-directed shRNA expression plasmid into SH-SY5Y cells, the cells were induced with $10 \mu \mathrm{M}$ RA for $2 \mathrm{~d}$. A twofold reduction in both primary (2.12) and secondary (2.17) neurite numbers was observed in the VCX knockdown cells relative to the control knockdown cells (Fig. 4B). A reduction in neurite numbers was also detected upon expression of the VCX-A ${ }^{\Delta \mathrm{N} 40}$ truncated protein in SH-SY5Y cells, suggesting the mutant VCX-A might function as a trans-dominant negative (Fig. 4C). However, expression of wild-type VCX-A into SH-SY5Y cells, which already express endogenous VCX proteins, had no appreciable consequence on differentiation with RA. Interestingly, regardless of changes in neurite numbers with the knockdown of VCX-A or expression of wild-type or truncated VCX-A, neurite length remained unchanged (supplemental Fig. S4, available at www.jneurosci.org as supplemental material), indicating that VCX-A is involved in the regulation of neurite number but not neurite length. Collectively, these data support a role for the VCX proteins as modulators of the number of neuronal cell projections.

\section{VCX-A can inhibit mRNA translation}

VCX-A is a noncanonical cap binding protein that is unable to bind the cap directly but only binds the cap when it is linked to an RNA (Jiao et al., 2006). This preferential binding to the $5^{\prime}$ end of 
an mRNA can lead to inhibition of decapping and stabilization of the mRNA (Jiao et al., 2006). The requirement for accessibility of the $5^{\prime}$ cap for mRNA translation in addition to its presence in staufen1containing RNA granules (Fig. 2G, $H$ ) also suggests that VCX-A could be a modulator of mRNA translation. Consistent with this hypothesis, addition of increasing concentrations of recombinant VCX-A to an in vitro mRNA translation reaction resulted in a dramatic inhibition of translation as determined by the level of luciferase activity (Fig. 5). In contrast, the VCX-A N-terminal truncation protein VCX-A ${ }^{\Delta \mathrm{N} 40}$, which is unable to bind the $5^{\prime}$ cap (Jiao et al., 2006), and an unrelated control protein UlP1 were unable to inhibit translation (Fig. 5A). We next tested whether VCX-A also inhibits protein translation in cells by assessing translation derived from a capped and polyadenylated firefly luciferase mRNA transfected into $293 \mathrm{~T}$ cells overexpressing either the FLAG-tagged VCX-A protein or control proteins. As shown in Figure 5B, translation was delayed in cells expressing FLAGtagged VCX-A compared with cells overexpressing the FLAG-VCX-A ${ }^{\Delta \mathrm{N} 40} \mathrm{mu}-$ tant protein or a control unrelated FLAGIKK protein. The decrease in luciferase activity was not due to a decrease in luciferase mRNA levels since, as expected, the stability of this mRNA increased in the presence of VCX-A in these assays (Fig. $5 C$ ). The above data indicate that VCX-A can function as a translational repressor both in vitro and in cells.

To further address the mechanism by which VCX-A can inhibit translation, we sought to identify cognate mRNAs that VCX-A might bind to and potentially regulate. The RNA-binding property of VCX-A (Jiao et al., 2006) indicates that it may specifically bind a subset of mRNA and regulate their expression. FLAGtagged VCX-A expressed in human SH-SY5Y neuroblastoma cells was immunopurified along with bound mRNA using a FLAG-M2 column. mRNAs copurifying with FLAGVCX-A were isolated and reverse transcribed into cDNAs. A small subset of mRNAs known to be modulators of neuritogenesis were tested for their potential selective binding by VCX-A. The mRNAs encoding two regulators of dendrite branching, glutamate receptor interacting protein 1 (GRIP1) and calcium/calmodulindependent protein kinase I (CaMKI), were bound by FLAG-VCX-A but not the germ line-specific mDAZL control protein (Fig. $6 A$ ). Three other mRNAs encoding proteins involved in neuritogenesis, RhoA,
A

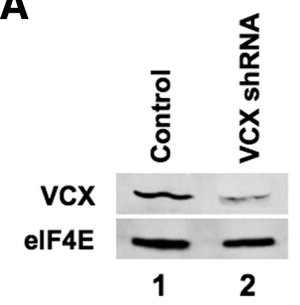

C

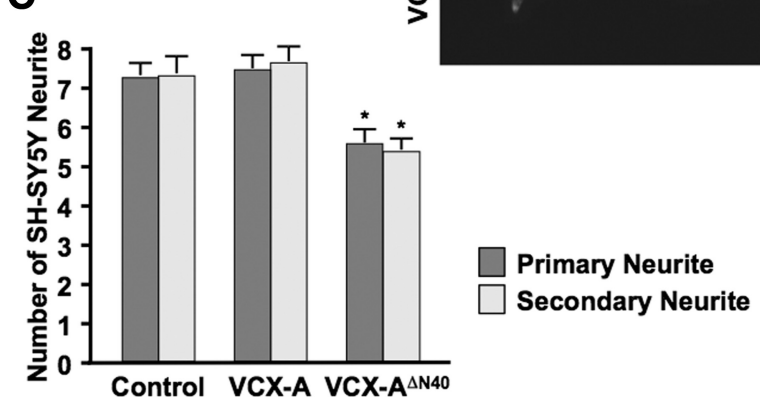

B
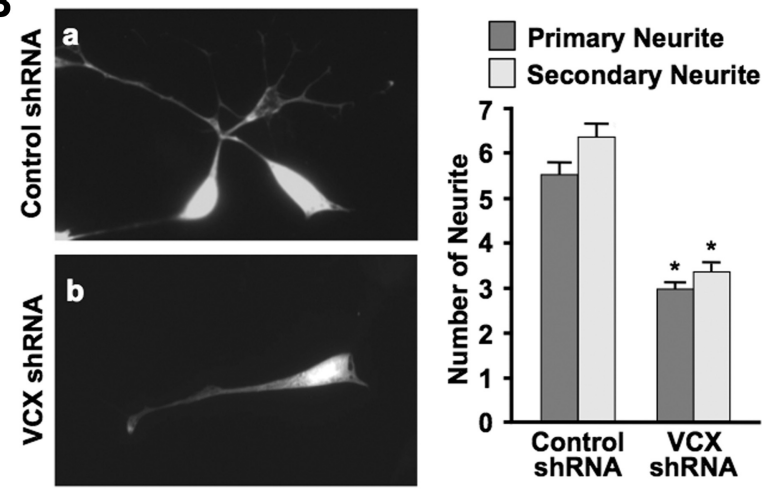

Figure 4. VCX affects proper neuritogenesis. Human SH-SY5Y neuroblastoma cells were transfected with plasmids expressing either VCX-directed shRNA to knockdown endogenous VCX levels, FLAG-tagged VCX-A, or VCX-A ${ }^{\triangle N 40}$ mutation plasmids to overexpress VCX-A. Differentiation was induced with $10 \mu \mathrm{m}$ retinoic acid for $2 \mathrm{~d}$. Transfected cells were identified and neurites were visualized by the coexpression of green fluorescent protein (GFP), detected with polyclonal anti-GFP antibody. $\boldsymbol{A}$, VCX protein levels were reduced following shRNA knockdown in SH-SY5Y cells detected by Western blot analysis with affinity-purified VCX-A antibody. elF4E was used as a protein loading control. $B$, A twofold reduction in both primary and secondary neurite numbers was observed in the VCX knockdown SH-SY5Y cells (2.67 and 3.13) relative to the control knockdown cells (5.67 and 6.8), respectively, in two independent experiments $\left({ }^{*} p<0.001, n=30\right.$; ANOVA followed by Tukey-Kramer multiple-comparisons test). GFP images of representative neurons cotransfected with the GFP expression plasmid with either the VCX-directed shRNA plasmid or control shRNA vector plasmid are indicated. C, Both primary and secondary neurite numbers were significantly reduced with overexpressed FLAG-VCX-A ${ }^{\Delta N 40}(5.60$ and 5.38) compared with transfected empty vector control cells (7.29 and 7.36) ( ${ }^{*} p<0.001, n \geq 59$; ANOVA followed by TukeyKramer multiple-comparisons test). Both primary and secondary neurite numbers were not significantly changed upon overexpression of FLAG-VCX-A, indicating that VCX-A levels are already saturating. Error bars represent \pm SD.
A

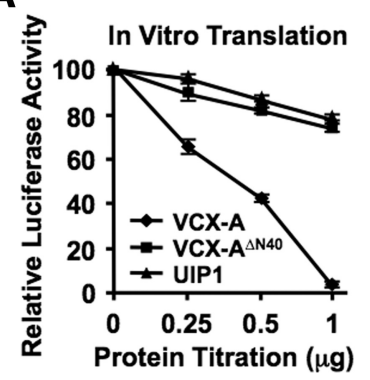

B

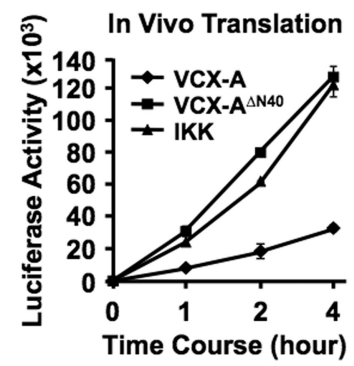

C

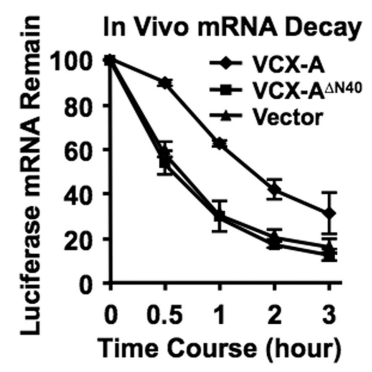

Figure 5. VCX-A inhibits protein translation in vitro and in cells. In vitro transcribed capped and polyadenylated firefly luciferase mRNA was used as substrate for protein translation. Translation efficiencies were measured by detecting luciferase activity. The assays were performed in triplicate. The data are represented as the mean and normalized values relative to assays lacking recombinant protein in $\boldsymbol{A}$ or to the initial zero time point in $\boldsymbol{B}$. $\boldsymbol{A}$, Firefly luciferase activity derived from the translation of luciferase mRNA in an in vitro rabbit reticulocyte lysate system in the presence of increasing titrations of His-tagged VCX-A, VCX-A ${ }^{\Delta 40}$ mutant unable to bind RNA, or control UIP1 recombinant protein is shown. VCX-A but not the VCX-A ${ }^{\Delta 40}$ mutant or UIP1 control proteins can dramatically inhibit luciferase mRNA translation in vitro. $B, 293$ T cells expressing FLAG-tagged VCX-A, VCX-A ${ }^{\Delta 40}$ mutant, or control IKK proteins were, electroporated with capped and polyadenylated firefly luciferase mRNA and luciferase activity was assessed from cells harvested at the indicated time points. Luciferase activity is presented relative to the zero time point and averaged from three independent experiments. Protein translation was significantly inhibited in cells expressing the VCX-A protein. $C$, Stability of the firefly luciferase mRNA electroporated into 293T cells is greater in cells stably expressing VCX-A ( $\left.t_{1 / 2}=95 \mathrm{~min}\right)$ than in the cells stably transformed with the VCX-A ${ }^{\Delta \mathrm{N} 40}$ truncation mutant or vector control $\left(t_{1 / 2}=37 \mathrm{~min}\right)$. Values were determined by quantitative realtime reverse transcriptase-PCR normalized to the GAPDH mRNA. Error bars represent \pm SD. 
A

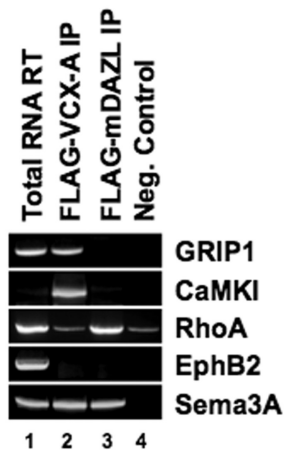

B

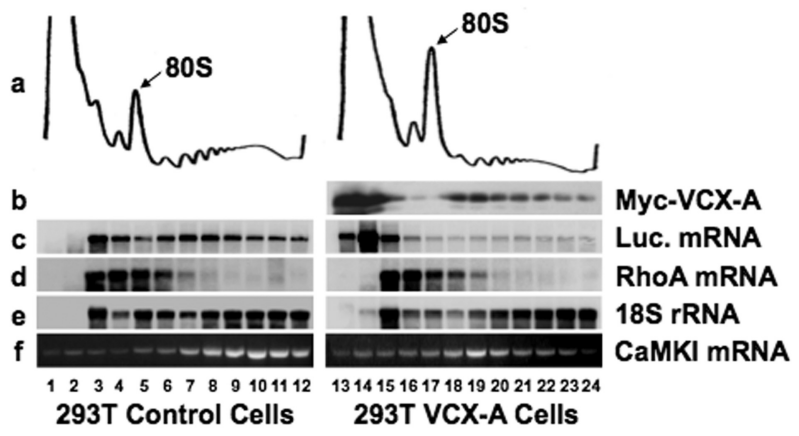

C

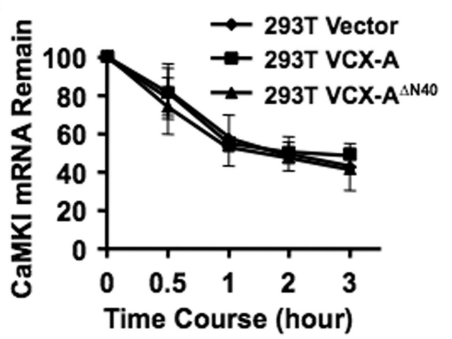

D

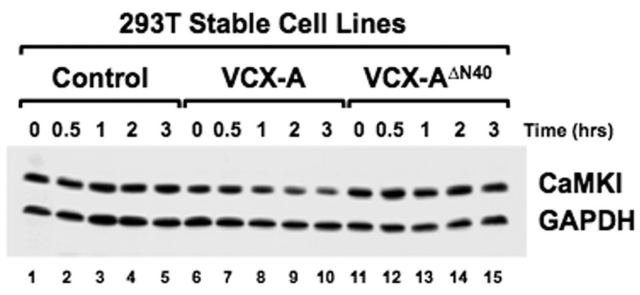

Figure 6. VCX-A shifts target mRNAs from high-density polysome fractions to mRNP fractions. $A, V C X-A$ can preferentially bind a subset of mRNAs. Human SH-SY5Y neuroblastoma cells transiently transfected with plasmids expressing FLAG-VCX-A or FLAGmDAZL control were induced to differentiate with $10 \mu \mathrm{m}$ retinoic acid $2 \mathrm{~d}$ after transfection and mRNAs copurifying with the FLAG-tagged proteins were isolated. The immunopurified mRNAs were oligo $\mathrm{d}(\mathrm{T})$ reverse transcribed and PCR amplified with transcript-specific primers. Products reverse transcribed from SH-SY5Y total RNA, FLAG-VCX-A coprecipitated RNA, FLAG-mDAZL coprecipitated RNA, or RNA without RT are as indicated. $B$, Polysome profiles were resolved through a $10-50 \%$ sucrose gradient prepared from 293T cells stably expressing VCX-A (right) or the empty vector control (left) and transiently transfected with firefly luciferase expression plasmid. The $80 \mathrm{~S}$ ribosomal complex is indicated (a). Protein and RNA from each fraction were separated by SDS-PAGE $(\boldsymbol{b})$, denaturing agarose gel electrophoresis $(\boldsymbol{c}-\boldsymbol{e})$, or nondenaturing agarose gel $(\boldsymbol{f})$. The location of epitope-tagged VCX-A protein was determined by Western analysis in $\boldsymbol{b}$. The RNAs in $\boldsymbol{c}-\boldsymbol{e}$ were detected by Northern blotting and the CaMKI mRNA distribution was detected by RT-PCR $(\boldsymbol{f})$. C, Stability of the CaMKI mRNA is unchanged upon overexpression of VCX-A. 293T cells stably transformed with myc-tagged VCX-A, VCX-A ${ }^{\Delta \mathrm{N} 40}$ truncation mutant, or control vector were harvested at the indicated time points following transcriptional arrest with actinomycin $D$ and endogenous CaMKI mRNA levels determined by quantitative RT-PCR normalized to the GAPDH mRNA. The results of three independent assays are shown with \pm SD denoted by the error bars. $D$, Levels of CaMKI protein are lower in cells expressing VCX-A. Protein extract from cells in C were used to detect CaMKI protein levels by Western blot analysis as indicated. GAPDH was used as protein loading control.

EphB2, and Sema3A, were not specifically bound by FLAG-VCX-A (Fig. 6A, compare lane 2 with lane 3). These data demonstrate that VCX-A can specifically bind CaMKI and GRIP1 mRNAs. The binding to CaMKI is noteworthy as this is a low-abundance mRNA that is not detected in the total RNA lane but is nonetheless selectively enriched by its association with FLAG-VCX-A.

To assess whether there is a functional significance for the binding of VCX-A, polysome profiles were analyzed in cells overexpressing VCX-A. Extract obtained from a $293 \mathrm{~T}$ cell line containing a stably integrated VCX-A gene or a control vector were generated and used. A clear increase in $80 \mathrm{~S}$ monosomes and a corresponding decrease in polysomes are apparent in cells overexpressing VCX-A relative to control cells (Fig. $6 \mathrm{Ba}$ ), consistent with a translational inhibition property for VCX-A. An increase in the $80 \mathrm{~S}$ population is indicative of a block in translation initiation. Western blot analysis revealed that exogenous mycVCX-A protein was predominantly at the top of the polysome profile gradient within the free messenger ribonucleoprotein particle (mRNP) fractions (Fig. $6 B b$, lanes 13,14 ) and residual amounts within the polysome fractions. We next tested the mRNA distribution of an exogenously transfected firefly luciferase mRNA whose translation was inhibited by VCX-A in vitro (Fig. 5) as well as the endogenous CaMKI mRNAs within the polysome distribution. Northern blot analysis revealed that the silenced exogenous firefly luciferase mRNA was shifted from the polysomal fractions in the control cells to the mRNP fractions in the presence of myc-VCX-A (Fig. 6Bc, compare lanes 7-12 with lanes 19-24 and lanes 1 and 2 with lanes 13 and 14). The redistribution of mRNAs from polysomes toward monosomes was also observed with the CaMKI mRNA detected by RT-PCR in the presence of overexpressed VCX-A protein (Fig. 6Bf). The effect of myc-VCX-A on translational silencing is relatively specific as shown by the fact that the distribution of RhoA mRNA was not detectably altered with an increase in VCX-A protein (Fig. $6 B d$ ). These data suggest that VCX-A may function to modulate mRNA translation.

To directly test the stability and translation of the CaMKI mRNA in cells, 293T cells were treated with actinomycin D to block transcription and CaMKI protein and mRNA levels were followed over time. Endogenous CaMKI mRNA has an approximate 80 min half-life in $293 \mathrm{~T}$ cells, which remained constant in the presence of overexpressed VCX-A or VCX-A ${ }^{\Delta \mathrm{N} 40}$ truncated protein (Fig. $6 \mathrm{C}$ ), as did the steady-state level of CaMKI mRNA in the different cells (data not shown). This is in contrast to the increased stability of the transfected luciferase mRNA upon overexpression of VCX-A (Fig. 5C), suggesting a potential transcript-specific role of VCX-A in mRNA stability. However, consistent with the polysome profile, CaMKI protein levels were lower in cells overexpressing VCX-A compared with the controls (Fig. 6D). Collectively, our data support a role for VCX-A in proper neuritogenesis and provide insight into a possible role for these proteins in cognitive function by the posttranscriptional control of gene expression.

\section{Discussion}

VCX-A was recently shown to be a member of a growing list of noncanonical cap binding proteins capable of simultaneously binding the $5^{\prime}$ cap and RNA, and as such, can modulate mRNA decapping (Jiao et al., 2006). We now demonstrate that VCX-A is also a regulator of mRNA translation and its capacity to bind the 5 ' end of an mRNA promotes inhibition of mRNA translation initiation. These properties of VCX-A suggest that it has the potential to bind a subset of mRNAs and stabilize and silence their expression. The positive effect of VCX-A in neurite outgrowth combined with the translational silencing by VCX-A suggests that it could modulate a subset of mRNAs encoding regulators of neuritogenesis.

VCX-A is implicated in X-linked mental retardation (Fukami et al., 2000) but was reported to be testis specific (Fukami et al., 2000; Lahn and Page, 2000; Zou et al., 2003). Indeed, by RT-PCR, the Y chromosome-localized VCY genes are exclusively and robustly expressed in male germ cells (Fig. 1A). Despite the reported testis germ cell restriction of the VCX genes, we initially cloned the cDNA of VCX-A from human K562 erythroleukemia 
cells (Jiao et al., 2006). The presence of the VCX-A mRNA in erythroleukemia cells indicated that expression of this gene is not restricted to testis. Western blot and RT-PCR analyses demonstrated that the VCX genes are expressed in all human tissues and cell lines we have tested thus far, including human brain (Fig. 1; supplemental Fig. S1, available at www.jneurosci.org as supplemental material). VCX proteins are also expressed in human neurons as determined by immunohistochemistry of postmortem human brain hippocampal and cortical sections (Fig. 1). Collectively, these data strongly indicate that the VCX genes are ubiquitously expressed in human tissues, and notably in neurons, consistent with a role in cognitive function. As the VCX/Y genes are only present in simian primates (Fukami et al., 2000; Lahn and Page, 2000) it is plausible that they appeared more recently in evolution. We also failed to identify a homologous protein or nucleic acid sequence in mouse or rat via the mining of currently available databases. Although an obvious homolog is not detectable, we cannot rule out the presence of a functional homolog in other mammalian organisms.

The presence of VCX proteins in discrete foci that colocalize with staufen 1-containing RNA granules suggests that these might be sites of VCX proteins in transit with silenced mRNAs. The preferential binding property of VCX-A to the $5^{\prime}$ end of mRNAs can both lead to stabilization of an mRNA (Fig. $5 C$ ) as well as inhibition of translation (Figs. 5, 6) are consistent with this possibility. Interestingly, although VCX-A has the potential to stabilize an mRNA (Fig. 5C), this function appears to be transcript specific, as it did not stabilize the CaMKI mRNA (Fig. 6C), suggesting a complex array of functions. At present, whether the mRNA stabilizing or translational silencing functions of VCX more significantly contribute to neuritogenesis is not known, but it is likely that a combination of both properties will be involved. The simultaneous function of both of these events could lead to assembly of mRNAs into stabilized and translationally silenced mRNPs.

Three important observations demonstrate a role for the VCX proteins in neurite number and provide insight into its potential role in cognitive function. First, the enhanced presence of VCXfoci within neurites following induction of SH-SY5Y cells by RA (Fig. 2) suggests a function during neurite development. Second, a reduction in neurite outgrowth in RA-induced SH-SY5Y cells with reduced VCX protein levels (Fig. $4 B$ ) underscores the significance of VCX proteins in this process. Last, overexpression of VCX-A in primary rat hippocampal neurons, which normally do not express the VCX genes, results in an increase in neurite number (Fig. 3), also demonstrating a direct correlation between the VCX proteins and neuronal processes.

The stimulation of neuronal processes by VCX implies that VCX either positively or negatively regulates mRNAs encoding proteins involved in neurite number and complexity. Considering the ability of VCX-A to stabilize mRNA (Fig. 5C) (Jiao et al., 2006) and silence mRNA translation (Figs. 5, 6), either or both of these properties could contribute to neuritogenesis. At present, it is not clear whether the VCX-A-mediated regulation of neurite outgrowth involves one specific or multiple substrate mRNAs, and a global analysis of VCX-A target substrates will be required to directly address this question. However, the preferential binding by VCX-A and the potential silencing of the mRNA encoding CaMKI (Fig. 6), a positive regulator of dendrite outgrowth (Kamata et al., 2007), and its colocalization with staufen1containing RNA granules, suggests that VCX-A could be involved in the transport of a subset of translationally silenced mRNAs that promote neuronal projections upon induction. Several RNA- binding proteins have been shown to regulate the translation of specific mRNAs that contribute to neuronal plasticity (Wu et al., 1998; Huber et al., 2002; Si et al., 2003; Alarcon et al., 2004; Huang et al., 2005; Koekkoek et al., 2005). Aberrant translation of mRNAs in neuronal cells can contribute to disease states, as is the case with Fragile X syndrome. This disorder is caused by the absence of, or a mutation in, the FMRP RNA-binding protein, which leads to misregulation of translation at synapses (Zalfa et al., 2006). Based on the modest expression level of the VCX proteins in brain, it is possible that this family of proteins function in transcript-specific regulation of mRNA that can contribute to neuritogenesis.

The ubiquitous presence of VCX in all tissues tested indicates that VCX also contributes to an mRNA stability and translational regulatory role in non-neuronal cells. Identification of mRNAs specifically bound by VCX in neuronal and non-neuronal cells will begin to address this model. An interesting question remains regarding regulation of the VCX association at the $5^{\prime}$ end of an mRNA. One possible regulatory role could be analogous to the Y-box protein, YB-1. YB-1, like VCX, is a noncanonical cap binding protein that preferentially binds the $5^{\prime}$ end of an mRNA to stabilize and silence an mRNA (Evdokimova et al., 2001). Its association with the $5^{\prime}$ cap is modulated by Akt-mediated phosphorylation, which reduces the affinity of YB-1 for the $5^{\prime}$ cap, resulting in the release of translational suppression (Evdokimova et al., 2006). Whether a similar mechanism is involved in the regulation of VCX binding to the $5^{\prime}$ cap remains to be determined.

An interesting observation from our studies is, translation of some, but not all, mRNAs tested were affected by VCX overexpression. It appears unlikely that all the affected RNAs have a specific primary sequence that VCX binds to. A more likely possibility is that VCX preferentially binds to a higher order structure at the mRNA 5' -end analogous to the Dcp2 decapping enzyme, a protein that similar to VCX, preferentially binds the $5^{\prime}$ end of mRNAs (Li et al., 2008). Collectively, our studies demonstrate that VCX-A, and most likely all members of the VCX/Y family of proteins, are modulators of mRNA stability and translation. They can preferentially bind the $5^{\prime}$ cap of an mRNA and potentially sequester the mRNA into a silenced mRNP complex to prevent its expression into protein. A decrease in VCX protein expression in neurons results in the reduction of neurite numbers but not length, suggesting that VCX is essential for the number of neuronal projections rather than their extension, by selectively modulating the expression of mRNAs involved in neurite outgrowth. Our data indicates that the origins of at least part of the implicated mental retardation that accompanies VCX-A deficiency could be a consequence of aberrant translation of a subset of mRNAs. Studies are underway to determine what mRNAs are specifically regulated by VCX and how this regulation contributes to neuronal differentiation.

\section{References}

Akum BF, Chen M, Gunderson SI, Riefler GM, Scerri-Hansen MM, Firestein BL (2004) Cypin regulates dendrite patterning in hippocampal neurons by promoting microtubule assembly. Nat Neurosci 7:145-152.

Alarcon JM, Hodgman R, Theis M, Huang YS, Kandel ER, Richter JD (2004) Selective modulation of some forms of Schaffer collateral-CA1 synaptic plasticity in mice with a disruption of the CPEB-1 gene. Learn Mem 11:318-327.

Busser J, Geldmacher DS, Herrup K (1998) Ectopic cell cycle proteins predict the sites of neuronal cell death in Alzheimer's disease brain. J Neurosci 18:2801-2807.

Chen H, Firestein BL (2007) RhoA regulates dendrite branching in hippocampal neurons by decreasing cypin protein levels. J Neurosci 27:83788386. 
Cuevas-Covarrubias SA, González-Huerta LM (2008) Analysis of the VCX3A, VCX2 and VCX3B genes shows that VCX3A gene deletion is not sufficient to result in mental retardation in X-linked ichthyosis. Br J Dermatol 158:483-486.

de Wet JR, Wood KV, DeLuca M, Helinski DR, Subramani S (1987) Firefly luciferase gene: structure and expression in mammalian cells. Mol Cell Biol 7:725-737.

Evdokimova V, Ruzanov P, Anglesio MS, Sorokin AV, Ovchinnikov LP, Buckley J, Triche TJ, Sonenberg N, Sorensen PH (2006) Akt-mediated YB-1 phosphorylation activates translation of silent mRNA species. Mol Cell Biol 26:277-292.

Evdokimova V, Ruzanov P, Imataka H, Raught B, Svitkin Y, Ovchinnikov LP, Sonenberg N (2001) The major mRNA-associated protein YB-1 is a potent $5^{\prime}$ cap-dependent mRNA stabilizer. EMBO J 20:5491-5502.

Firestein BL, Firestein BL, Brenman JE, Aoki C, Sanchez-Perez AM, El-Husseini AE, Bredt DS (1999) Cypin: a cytosolic regulator of PSD-95 postsynaptic targeting. Neuron 24:659-672.

Frey U, Krug M, Reymann KG, Matthies H (1988) Anisomycin, an inhibitor of protein synthesis, blocks late phases of LTP phenomena in the hippocampal CA1 region in vitro. Brain Res 452:57-65.

Fukami M, Kirsch S, Schiller S, Richter A, Benes V, Franco B, Muroya K, Rao E, Merker S, Niesler B, Ballabio A, Ansorge W, Ogata T, Rappold GA (2000) A member of a gene family on Xp22.3, VCX-A, is deleted in patients with X-linked nonspecific mental retardation. Am J Hum Genet 67:563-573.

Garneau NL, Wilusz J, Wilusz CJ (2007) The highways and byways of mRNA decay. Nat Rev Mol Cell Biol 8:113-126.

Grossman AW, Elisseou NM, McKinney BC, Greenough WT (2006) Hippocampal pyramidal cells in adult Fmrl knockout mice exhibit an immature-appearing profile of dendritic spines. Brain Res 1084:158-164.

Hosomi N, Oiso N, Fukai K, Hanada K, Fujita H, Ishii M (2007) Deletion of distal promoter of VCXA in a patient with X-linked ichthyosis associated with borderline mental retardation. J Dermatol Sci 45:31-36.

Huang CS, Shi SH, Ule J, Ruggiu M, Barker LA, Darnell RB, Jan YN, Jan LY (2005) Common molecular pathways mediate long-term potentiation of synaptic excitation and slow synaptic inhibition. Cell 123:105-118.

Huber AB, Weinmann O, Brösamle C, Oertle T, Schwab ME (2002) Patterns of Nogo mRNA and protein expression in the developing and adult rat and after CNS lesions. J Neurosci 22:3553-3567.

Jiao X, Wang Z, Kiledjian M (2006) Identification of an mRNA-decapping regulator implicated in X-linked mental retardation. Mol Cell 24:713722.

Kamata A, Sakagami H, Tokumitsu H, Owada Y, Fukunaga K, Kondo H (2007) Spatiotemporal expression of four isoforms of Ca2+/calmodulin-dependent protein kinase I in brain and its possible roles in hippocampal dendritic growth. Neurosci Res 57:86-97.

Kiebler MA, Bassell GJ (2006) Neuronal RNA granules: movers and makers. Neuron 51:685-690.

Koekkoek SK, Yamaguchi K, Milojkovic BA, Dortland BR, Ruigrok TJ, Maex R, De Graaf W, Smit AE, VanderWerf F, Bakker CE, Willemsen R, Ikeda T, Kakizawa S, Onodera K, Nelson DL, Mientjes E, Joosten M, De Schutter E, Oostra BA, Ito M, et al. (2005) Deletion of FMR1 in Purkinje cells enhances parallel fiber LTD, enlarges spines, and attenuates cerebellar eyelid conditioning in Fragile X syndrome. Neuron 47:339-352.

Lahn BT, Page DC (2000) A human sex-chromosomal gene family ex- pressed in male germ cells and encoding variably charged proteins. Hum Mol Genet 9:311-319.

Li Y, Song MG, Kiledjian M (2008) Transcript-specific decapping and regulated stability by the human Dcp2 decapping protein. Mol Cell Biol 28:939-948.

Liu H, Kiledjian M (2006) Decapping the message: a beginning or an end. Biochem Soc Trans 34:35-38.

Liu SW, Jiao X, Liu H, Gu M, Lima CD, Kiledjian M (2004) Functional analysis of mRNA scavenger decapping enzymes. RNA 10:1412-1422.

Livak KJ, Schmittgen TD (2001) Analysis of relative gene expression data using real-time quantitative PCR and the 2(-Delta Delta C(T)) Method. Methods 25:402-408.

Macarov M, Zeigler M, Newman JP, Strich D, Sury V, Tennenbaum A, Meiner V (2007) Deletions of VCX-A and NLGN4: a variable phenotype including normal intellect. J Intellect Disabil Res 51:329-333.

McKinney BC, Grossman AW, Elisseou NM, Greenough WT (2005) Dendritic spine abnormalities in the occipital cortex of C57BL/6 Fmr1 knockout mice. Am J Med Genet B Neuropsychiatr Genet 136B:98-102.

Nguyen PV, Abel T, Kandel ER (1994) Requirement of a critical period of transcription for induction of a late phase of LTP. Science 265:1104-1107.

Paddison PJ, Hannon GJ (2002) RNA interference: the new somatic cell genetics? Cancer Cell 2:17-23.

Påhlman S, Ruusala AI, Abrahamsson L, Mattsson ME, Esscher T (1984) Retinoic acid-induced differentiation of cultured human neuroblastoma cells: a comparison with phorbolester-induced differentiation. Cell Differ 14:135-144.

Rodgers ND, Jiao X, Kiledjian M (2002) Identifying mRNAs bound by RNA-binding proteins using affinity purification and differential display. Methods 26:115-122.

Si K, Giustetto M, Etkin A, Hsu R, Janisiewicz AM, Miniaci MC, Kim JH, Zhu $\mathrm{H}$, Kandel ER (2003) A neuronal isoform of CPEB regulates local protein synthesis and stabilizes synapse-specific long-term facilitation in Aplysia. Cell 115:893-904.

Sutton MA, Schuman EM (2005) Local translational control in dendrites and its role in long-term synaptic plasticity. J Neurobiol 64:116-131.

Van Esch H, Hollanders K, Badisco L, Melotte C, Van Hummelen P, Vermeesch JR, Devriendt K, Fryns JP, Marynen P, Froyen G (2005) Deletion of VCX-A due to NAHR plays a major role in the occurrence of mental retardation in patients with X-linked ichthyosis. Hum Mol Genet 14:1795-1803.

Wang Z, Day N, Trifillis P, Kiledjian M (1999) An mRNA stability complex functions with poly(A)-binding protein to stabilize mRNA in vitro. Mol Cell Biol 19:4552-4560.

Wang Z, Jiao X, Carr-Schmid A, Kiledjian M (2002) The hDcp2 protein is a mammalian mRNA decapping enzyme. Proc Natl Acad Sci USA 99:12663-12668.

Wu L, Wells D, Tay J, Mendis D, Abbott MA, Barnitt A, Quinlan E, Heynen A, Fallon JR, Richter JD (1998) CPEB-mediated cytoplasmic polyadenylation and the regulation of experience-dependent translation of alphaCaMKII mRNA at synapses. Neuron 21:1129-1139.

Zalfa F, Achsel T, Bagni C (2006) mRNPs, polysomes or granules: FMRP in neuronal protein synthesis. Curr Opin Neurobiol 16:265-269.

Zou SW, Zhang JC, Zhang XD, Miao SY, Zong SD, Sheng Q, Wang LF (2003) Expression and localization of VCX/Y proteins and their possible involvement in regulation of ribosome assembly during spermatogenesis. Cell Res 13:171-177. 